\title{
Spacetime as a New Frontier Advanced Material with Applications in Physics, Engineering, Chemistry and Cosmology*
}

\author{
Mohamed S. El Naschie \\ Department of Physics, Faculty of Science, University of Alexandria, Alexandria, Egypt \\ Email: chaossf@aol.com
}

How to cite this paper: El Naschie, M.S. (2017) Spacetime as a New Frontier Advanced Material with Applications in Physics, Engineering, Chemistry and Cosmology. Advances in Materials Physics and Chemistry, 7, 347-352.

https://doi.org/10.4236/ampc.2017.79027

Received: August 9, 2017

Accepted: September 11, 2017

Published: September 14, 2017

Copyright $\odot 2017$ by author and Scientific Research Publishing Inc. This work is licensed under the Creative Commons Attribution International License (CC BY 4.0). http://creativecommons.org/licenses/by/4.0/

\section{Keywords}

N Tesla's Aether, Einstein's Aether

The starting point of the present work is a theoretical and experimental resolution of one of the most basic fundamental problems of physics, namely the very existence and reality of the Aether [1]-[11]. These basic philosophical and scientific insightful results in theoretical physics and cosmology [1]-[24] are converted here into a serious attempt to actually realizing an old futuristic dream, namely obtaining free energy from empty space or spacetime singularities as thought of by visionaries such as N. Tesla and Sir R. Penrose [2] [5], with undreamed of possibilities in physics, chemistry, engineering and cosmology [25]-[31].

Our mathematical and indirect experimental verdict which was announced recently [29] is that the Aether exists and it can be equated to the empty set of pure mathematics [3] [12] [13] [14] [15] [16]. More precisely the Aether may be understood for all mathematical and physical purposes as being identical to the empty set [8]-[14] underlying the Penrose fractal tessellation universe [17] which obeys the A. Connes' corresponding dimensional function of his noncommutative geometry [18] [19] [20] [21].

From this astounding conclusion it is relatively very easy to conclude from the above that cosmic dark energy is simply the energy stored in the Aether empty set [22] [23] [24] so that the agreement between the theoretical calculation and the numerous accurate cosmological measurements may be taken as an indirect experimental confirmation of this spectacular model for the Aether [10]-[16],

${ }^{\star}$ Dedicated to the memory of Mae-Wan Ho (1941-2016). A great scientist and a great lady. 
[22] [23] [24] In fact the mere fact that Penrose fractal tessellation is a well known and valid model for quasi crystals, i.e. a real material with five fold symmetry as well as for the cosmos at large [10] [17], is a sufficient fact to reinvigorate an old analogy between photons and phonon [7]-[11]. Not only that but far more importantly, one can look for methods of harnessing the energy of empty spacetime using nanotechnology [25]-[31] as a scientific fact, not fiction.

Seen in this larger imaginative new reality [7], we can go on confidently repeating what we have claimed for many years, that spacetime is physically real and may be regarded as a highly advanced material which can be used to yield practically infinite clean, free energy via what we called a dark energy-Casimir nanotech reactor [15]. As we said earlier on, such a reactor was thought of by many visionaries before but was never given anything like a firm mathematical and experimental justification as we did within the E-infinity theory proposal [21]-[36]. In fact based on these ideas two of the most active researchers in the Romanian chapter of fractal Cantorian spacetime realized as early as in 2007 that nanotechnology is the tool to construct a spacetime Casimir energy reactor [30].

We leave it to the historians of science to relate our findings to that of the Tesla-Einstein discussion about the Aether [5]. However it is interesting to note that while Einstein started by thinking of his theory of relativity as a proof that the Aether could not exist or at a minimum is a totally unnecessary assumption, in his later years Einstein modified his stance and came much nearer to the thinking of legendary Serbian-American N. Tesla who was a firm believer in the reality of the Aether [2] [5] [6] [7].

In what follows and in spite of the limited space of the present work we should and will give at least some mathematical elaboration in addition to the most important equations of our theory [8]-[36]. Proceeding in this way it will become evident that $E=m c^{2}$ of Einstein implicitly included a recognition of the existence of the Aether by unconsciously including the energy of the Aether, i.e. a totally empty spacetime via the energy of the quantum wave as explained by the author on many previous occasions [3] [4] [9]-[18] [34] [35] [36].

This conclusion, as easily reasoned, is a natural consequence of modelling the quantum pre-particle by the zero set [3] [6] [9] [11] and assign to it two dimensions, namely the topological dimension zero as befitting a point particle and the second Hausdorff dimension $\phi=(\sqrt{5}-1) / 2$ as obvious from Sir R. Penrose geometrical tessellation and Prof. A. Connes' dimensional function of the Penrose fractal universe [11] [17] [19] [20]. The pre-quantum wave on the other hand is interpreted in our theoretical model as the cobordism, i.e. the surface of the pre-particle [11]-[16]. Consequently it is an empty set and possesses two dimensions, similar to the zero set [4] [8]-[15]. The first is the Menger-Urysohn dimension minus one and the second is the Hausdorff dimension $\phi^{2}$ [11]-[20]. Thus following the rationale of our theory we see that the cobordism of the pre-quantum wave must be given by a topological dimension minus two and a Hausdorff dimension $\phi^{3}$ [10] [11]. On the other hand the average Hausdorff 
dimension of spacetime is $4+\phi^{3}$ [10] [11] [21]. Consequently the inverse of $4+\phi^{3}$ is $\phi^{3}$ [12] [13] [14] [29]-[36]. That means on average the surface of the pre-quantum wave is spacetime itself [12] [13]. That way we may see the quantum particle with its surrounding guiding pre-quantum wave as ripples in spacetime just as phonon are vibrational ripples in the quasi crystal [7]-[16]. Taking a bird's eye view of the entire situation, Aether, quantum field, quantum wave and spacetime become different names for very similar, in fact almost identical physico-mathematical entities [10]-[24]. Since spacetime is an empty set then deep philosophical implications with a bearing in both physics and metaphysics as well scientific philosophy follows. Thus whenever a zero set particle moves the surrounding empty set becomes a non-empty zero set. This is a self refferential statement which implies fractals [35]. It also implies zenon paradox about motion being an illusion [36].

It remains only to quantify the situation. Putting the five fold quasi crystal symmetry which was thought not long ago to be forbidden [37] [38] in a Kaluza-Klein five dimensional manifold we find a five dimensional zero set topological volume $\phi^{5}$ [16]-[21]. Thus the surface of $\phi^{5}$ is clearly an additive five dimensional area equal $5 \phi^{2}$ representing the pre-quantum wave [3] [4] [10]-[16]. Now we have shown in many previous publications [10]-[16] that $\phi^{5}$ is related to the ordinary measurable energy density of the universe and is found from the ordinary formula of classical kinetic energy when we let the velocity tend to that of light and find that $E(O)=(1 / 2)\left(m=\phi^{5}\right)\left(c^{2}\right)$ where $c=\phi$ is the topological speed of light which amounts to about $4.5 \%$ of the energy density of special relativity, namely $E=m c^{2}$ divided by 22 [10]-[16] [22] [23] [24]. As for the pre-quantum wave which is what gives us the dark energy sector and which cannot be measured directly because an empty set becomes non-empty and collapses on measurement [10]-[16] [22] [23] [24], one finds $E(D)=(1 / 2)(m=5)\left(c^{2}\right)$ which amounts to $95.5 \%$ of Einstein's density [12]-[24] that is approximately equal to Einstein's maximal density $\mathrm{mc}^{2}$ multiplied by 21 and divided again by 22 .

The beauty of the present theory is summarized in unexpected confirmation of Einstein's famous formula, namely [22]-[24]

$$
\begin{aligned}
E & =E(O)+E(D) \\
& =\left[\left(\phi^{5} / 2\right) m c^{2}+\left(5 \phi^{2} / 2\right) m c^{2}\right] \\
& =(1 / 2)\left(\phi^{5}+5 \phi^{2}\right) m c^{2} \\
& =(1 / 2)(2) m c^{2} \\
& =E(\text { Einstein })
\end{aligned}
$$

Note that the most important point in all of the above results is in complete agreement with the accurate cosmic measurements [11]-[16] [22] [23] [24]. Consequently our theory is an accurate model of reality [21]-[36].

Finally the present theory may be refined to account for dark matter energy and pure dark energy as well [23] [24]. That way we can rewrite Equation (1) 
using the fractal Kaluza-Klein spacetime theory which replaces 5 by $5+\phi^{3}$ as the sum of not two but three energy sectors densities as follows:

$$
E+E_{1}+E_{2}+E_{3}
$$

where [22] [23] [24] [28] [29]

$$
\begin{aligned}
E_{1} & =E(O) \\
& =\left(\frac{\phi^{3}}{5+\phi^{3}}\right) m c^{2}=0.04508497178 m c^{2}
\end{aligned}
$$

while $E_{2}$ is the energy density of dark matter in the universe given by

$$
E_{2}=\left(\frac{1+\Delta}{5+\phi^{3}}\right)\left(m c^{2}\right)=0.2218033994 m c^{2}
$$

and $\Delta=0.1613776766$ is a coupling constant as explained elsewhere [28] [29]. Finally $E_{3}$ is the pure dark energy in the universe and is given by [22] [23] [24] [28] [29]

$$
E_{3}=\left(\frac{4-\Delta}{5+\phi^{3}}\right)\left(m c^{2}\right)=0.7331116289 m c^{2}
$$

It was the great German material scientist and physicist at Clausthal University who noticed first explicitly that the ordinary energy density when inversed gives the exact dark matter percentage [31]. This is of course implicitly understood from the E-infinity relations between the various fundamental equations of the golden mean harmony [32] [33] [34].

From the preceding result we see that there is no doubt that the Aether exists and that it is basically a five dimensional empty set akin to the Penrose tessellation universe which is described by A. Connes' dimensional function of his noncommutative geometry as we indicated earlier on [17] [18] [19].

As for the actual design of the reactor, we stress yet again this is actually a nanotechnology problem of restructuring empty space as we indicated earlier on and is basically building nano universes using nano fullerene buckyballs [39] particles in a way not dissimilar to what we discussed in some preliminary design proposals (see for instance Fig. 4 of Ref. [25]).

In conclusion, on an optimistic note, we should be glad to see that we have come a long way from Tesla's dreams [5] to a Nobel winner in physics saying that spacetime is a material [7] and from forbidden 5 fold symmetry to a Nobel Prize for quasi crystals and buckyballs [37] [38] [39].

\section{References}

[1] Penrose, Sir.R. (1991) The Mass of the Classical Vacuum. In: Saunders, S. and Browen, H., Eds., The Philosophy of The Vacuum, Clarendon Press, Oxford University, Oxford, UK, 21-26.

[2] Einstein, A. (1922) Ether and the Theory of Relativity. 5th May 1920 at the University of Leiden, Holland. Methuen \& Co. Ltd., London.

http://www-history.mcs.st-andrews.ac.uk/Extras/Einstein_ether.html 
[3] Marek-Crnjac, L. (2013) Cantorian Space-Time Theory: The Physics of Empty Sets in Connection With Quantum Entanglement and Dark Energy. Lambert Academic Publishing, Saarbrucken.

[4] El Naschie, M.S. (2011) On the Philosophy of Being and Nothingness in Fundamental Physics. Nonlinear Science Letters B, 1, 4-5.

[5] Seifer, M.J. (2009) Tesla vs Einstein and the Birth of the New Physics. New Dawn No. 113 (March-April).

http://www.newdawnmagazine.com/articles/tesla-vs-einstein-the-ether-the-birth-of -the-new-physics

[6] Einstein, A. Einstein Relativity Theory Declares Aether Necessary (Youtube). https://www.youtube.com/watch?v=yH9vAIdMqng

[7] Wliczek, F. and Krauss, L. Materiality of a Vacuum (YouTube) https://www.youtube.com/Watch?V=BBXDrNn6PVg

[8] Auffray, J.-P. (2014) E-Infinity Dualities, Discontinuous Spacetimes, Xonic Quantum Physics and the Decisive Experiment. Journal of Modern Physics, 5, 1427-1436.

[9] Auffray, J.-P. (2015) E-infinity, the Zero Set, Absolute Space and the Photon Spin. Journal of Modern Physics, 6, 536-545. https://doi.org/10.4236/jmp.2015.65058

[10] Marek-Crnjac, L. (2015) On El Naschie’s Fractal-Cantorian Space-Time and Dark Energy-A Tutorial Review. Natural Science, 7, 581-598.

https://doi.org/10.4236/ns.2015.713058

[11] El Naschie, M.S. (2016) The Self Similarity Equivalence Relation Connecting Newton's Energy with Einstein's Energy and Dark Energy. International Journal of Innovation in Science and Mathematics, 4, 42-57.

[12] El Naschie, M.S. (2015) An Exact Mathematical Picture of Quantum Spacetime. Advances in Pure Mathematics, 5, 560-570. https://doi.org/10.4236/apm.2015.59052

[13] El Naschie, M.S. (2016) The Emergence of Spacetime from the Quantum in Three Steps. Advances in Pure Mathematics, 6, 446-454. https://doi.org/10.4236/apm.2016.66032

[14] El Naschie, M.S. (2016) On a Fractal Version of Witten's M-Theory. Journal of Astronomy \& Astrophysics, 6, 135-144.

[15] El Naschie, M.S. (2015) On a Non-Perturbative Quantum Relativity Theory Leading to a Casimir-Dark Energy Nanotech Reactor Proposal. Open Journal of Applied Science, 5, 313-324. https://doi.org/10.4236/ojapps.2015.57032

[16] El Naschie, M.S. (2017) Einstein-Kaluza Combined Spacetime as the Optimal and Simplest Framework to Compute and Understand Dark Matter, Pure Dark Energy and Measurable Ordinary Energy. Natural Science, 9, 241-244. https://doi.org/10.4236/ns.2017.98024

[17] El Naschie, M.S. (1998) Penrose Universe and Cantorian Spacetime as a Model for Noncommutative Quantum Geometry. Chaos, Solitons \& Fractals, 9, 931-933.

[18] El Naschie, M.S. (1998) Von Neumann Geometry and E-Infinity Quantum Spacetime. Chaos, Solitons \& Fractals, 9, 2023-2030.

[19] Connes (1994) Noncommutative Geometry. Academic Press, San Diego.

[20] El Naschie, M.S. (1994) On Certain "Empty" Cantor Sets and Their Dimensions. Chaos, Solitons \& Fractals, 4, 293-296.

[21] El Naschie, M.S. (2004) A Review of E-Infinity Theory and the Mass Spectrum of High Energy Particle Physics. Chaos, Solitons \& Fractals, 19, 209-236.

[22] El Naschie, M.S. (2017) Kähler Dark Matter, Dark Energy, Cosmic Density and 
Their Coupling. Journal of Modern Physics, 7, 1953-1962.

[23] El Naschie, M.S. (2017) A Combined Heterotic String and Kähler Manifold Elucidation of Ordinary Energy, Dark Matter, Olbers's Paradox and Pure Dark Energy Density of the Cosmos. Journal of Modern Physics, 8, 1101-1118. https://doi.org/10.4236/jmp.2017.87071

[24] El Naschie, M.S. (2017) From a Dual Einstein-Kaluza Spacetime to 'tHooft Renormalon and the Reality of Accelerated Cosmic Expansion. Journal of Modern Physics, 8, 1319-1329. https://doi.org/10.4236/jmp.2017.88085

[25] El Naschie, M.S. (2015) A Casimir-Dark Energy Nano Reactor Design Phase I. Natural Science, 7, 287-298. https://doi.org/10.4236/ns.2015.76032

[26] El Naschie, M.S. (2015) Casimir-Dark Energy Nano Reactor Design Proposal Based on Fractals. International Journal of Innovation is Science and Mathematics, 3, 187-194.

[27] El Naschie, M.S. (2015) A Cold Fusion-Casimir Energy Nano Reactor Proposal. World Journal of Nano Science and Engineering, 5, 49-56. https://doi.org/10.4236/wjnse.2015.52007

[28] El Naschie, M.S. (2015) Kerr Black Hole Geometry Leading to Dark Matter and Dark Energy via E-Infinity Theory and the Possibility of Nano Spacetime Singularity Reactor. Natural Science, 7, 210-225. https://doi.org/10.4236/ns.2015.74024

[29] El Naschie, M.S. (2017) The Aether of Spacetime Physics Is the Empty Set of Pure Mathematics. Natural Science.

[30] Agop, M., Stan, C., Tomas, M. ad Rusu, I.A. (2007) Fractal Spacetime Theory and Some Applications in Advanced Materials. Proceedings of the Romanian Academy, Series $A, \mathbf{8}$.

[31] Otto, H.H. (2017) Should We Pay More Attention to the Relationship between the Golden Mean and Archimedes' Constant. Nonlinear Science Letters A, 8, 410-412.

[32] El Naschie, M.S. (2008) Transfinite Harmonization by Taking the Dissonance out of the Quantum Field Symphony. Chaos, Solitons \& Fractals, 36, 781-786.

[33] El Naschie, M.S. (2017) Looped Light on Dark Energy. Journal of Quantum Information Science, 7, 43-47. https://doi.org/10.4236/jqis.2017.72004

[34] Ho, M.-W. (2017) Meaning of Life and the Universe: Transforming. World Scientific, Singapore.

[35] El Naschie, M.S. (2015) The Self Referential Pointless Universe Geometry as the Key to the Resolution of the Black Hole Information Paradox. International Journal of Innovation in Science and Mathematics, 3, 254-265.

[36] El Naschie, M.S. (2016) Cantorian-Fractal Kinetic Energy and Potential Energy as the Ordinary and Dark Energy Density Respectively. Natural Science, 8, 511-540. https://doi.org/10.4236/ns.2016.812052

[37] El Naschie, M.S. (1994) Forbidden Symmetries, Cantor Sets and Hypothetical Graphite. Chaos, Solitons \& Fractals, 4, 2269-2272.

[38] Malhotra, R. (2012) The Story of Nobel-Winning “Quasicrystals”. Current Science, 102, 1356-1357.

[39] Girifalco, L., Hodak, M. and Lee, R.S. (2000) Carbon Nanotubes, Balls, Ropes and a Universal Graphitic Potential. Physical Review B, 62, 13104-13114. https://doi.org/10.1103/PhysRevB.62.13104 
Submit or recommend next manuscript to SCIRP and we will provide best service for you:

Accepting pre-submission inquiries through Email, Facebook, LinkedIn, Twitter, etc. A wide selection of journals (inclusive of 9 subjects, more than 200 journals)

Providing 24-hour high-quality service

User-friendly online submission system

Fair and swift peer-review system

Efficient typesetting and proofreading procedure

Display of the result of downloads and visits, as well as the number of cited articles Maximum dissemination of your research work

Submit your manuscript at: http://papersubmission.scirp.org/

Or contact ampc@scirp.org 